\title{
Textual Article Clustering in Newspaper Pages
}

\author{
Marco Aiello \& Andrea Pegoretti \\ Dep. of Information and Communication Technologies \\ Università di Trento \\ Via Sommarive, 14 \\ 38100 Trento, Italy \\ aiellom@dit.unitn.it andpego@supereva.it
}

\begin{abstract}
In the analysis of a newspaper page an important step is the clustering of various text blocks into logical units, i.e., into articles. We propose three algorithms based on text processing techniques to cluster articles in newspaper pages. Based on the complexity of the three algorithms and experiment on actual pages from the Italian newspaper L'Adige, we select one of the algorithms as the preferred choice to solve the textual clustering problem.
\end{abstract}

\section{Introduction}

One of the first and most evident consequences of the revolution brought about by the diffusion of computers and by the great success of the Internet is a gradual decrease of the quantity of paper documents in our everyday life: we can read our favorite newspaper on the Internet; we can be informed minute by minute on the latest news by blogs and newsletters; we can read a book in electronic format. Nevertheless, there is a body of paper documents still circulating and being printed. These need to be converted into digital format for a number of reasons which include: digital storage, document retrieval, document transmission, document mutli-modal delivery (e.g., text-to-speech or visualization on handheld devices), document summarization, and so on.

The quantity of documents that needs to be converted into digital format is thus increasing, creating the need for systems capable to extracting knowledge and 'understanding' documents automatically. Such systems must be able not only to scan and store documents, but also to process the information contained within. On a printed document, these tasks must be performed by a human operator, which involves high costs in terms of work and time, and limited coverage of the document collections. 
The purpose of Document Image Analysis (DIA) is to recover information from paper documents, including not only the text but also images, layouts and all the components that characterize a printed document. There are various applications of DIA: from the digitalization of old books and newspapers to the automatic recognition of hand-compiled forms; from the automatic delivery of mail to the creation of text-to-speech systems capable of reading any kind of paper document to visually impaired people.

DIA is the set of techniques involved in recovering syntactic and semantic information from images of documents, mainly scanned versions of paper documents. The syntactic information is represented by the layout structure of the document, where each basic document object (i.e., indissoluble unit of text, titles, captions, figures, etc.) is identified and described by its content and its topological information (position in the page, width, height, etc.). The semantic information is collected into the logical structure comprising both the labeling of the document object (each document object is assigned a label indicating its role: title, sub-title, plain text, page number, etc.) and the detection of the reading order, i.e., the sequence of document objects the user is supposed to read.

\section{$1.1 \quad$ Related work}

Various systems have been proposed for document image analysis. A great body of work is dedicated to mail automation, form processing and processing of business letters, for instance [Cesarini et al., 1998, Dengel and Dubiel, 1997]. Lee [Lee et al., 2000] describes a system that analyzes journals from IEEE, specifically Transactions on Pattern Analysis and Machine Intelligence. Walischewski [Walischewski, 1997] presents a system to process business letters. The use of learning modules leads to more adaptable systems as those presented by Sainz and Dimitriadis [Palmero and Dimitriadis, 1999] and $\mathrm{Li}$ and $\mathrm{Ng}$ [ $\mathrm{Li}$ and $\mathrm{Ng}, 1999$ ]. These latter two systems ignore the important role of textual content. All the above systems are specific to some document class and consider documents with a unique reading order. Klink [Klink et al., 2000] uses textual features and geometrical relations for the classification of document objects, based on fuzzy-matched rules. The reading order detection problem is not addressed.

A prominent example of a system for processing newspaper pages is the one developed by Tsujimoto and Asada [Tsujimoto and Asada, 1992], which is tuned to process regular multicolumn black-and-white newspapers. For both layout and logical structure detection, domain knowledge is hard-coded into four transformation rules. The use of a tree based representation for the document restricts the class of documents that can be processed. In addition, the rules apply only to the specific document class and cannot be adapted to other classes. No use is made of the textual content of the articles. Furthermore, the system does not work for 
newspapers with very complex layouts.

\subsection{Three algorithms for article clustering}

In [Aiello et al., 2002] we presented a system which, by using spatial reasoning [Aiello and Smeulders, 2004] and text processing techniques [Sparck Jones, 1972, Salton and McGill, 1983, Baeza-Yates and Ribeiro-Neto, 1999], is able to extract the reading order from document images for documents having very different layouts. In particular, a spatial language for rectangles [Balbiani et al., 1998], based on the work on interval relations of Allen [Allen, 1983], is used to describe both the elements in a document and general rules to analyze them. In that work, the focus is on the heterogeneity of the document collection, and on the extraction of a unique reading order for each document image.

In this paper, we focus on newspaper pages, which have the additional problem that on each page there are several independent articles having independent reading orders. In addition, newspaper pages are an interesting application case since they are still widely used in paper format, and there are vast collections of newspapers archived in paper or microfilm form.

We propose three text processing-based algorithms for the problem of article clustering in newspaper pages, that is, the identification of text blocks which belong to the same article, and provide a complexity analysis of the algorithms. Via experimental results carried out on pages from the Italian regional newspaper L'Adige (http://www.ladige.it) we also show that text processing techniques work for the problem of article clustering, and compare the performances of the various algorithms. Surprisingly, the simplest algorithm has similar performance compared to the more sophisticated ones, and has significantly lower complexity, and it is therefore the one we propose as the algorithm of choice.

The reminder of the paper is organized as follows. In Section 2, we state the article clustering problem and provide three algorithms to address it. In Section 3 we provide experimental results and a detailed discussion. In Section 4, we provide a complexity analysis of the three algorithms. Conclusions are presented in Section 5.

\section{Article Clustering Algorithms}

Newspaper pages are generally formed from several independent articles which are scattered throughout the page in columns. For simple pages, a human reader can determine the division into articles and the reading order from layout and typesetting information alone, but in the case of complex newspaper pages the only way to understand the page is to read the text and compare the contents of the blocks. Consider the sample page from $L$ 'Adige in Figure 1 where 
the running text blocks are highlighted by black rectangles. Some articles are easy to separate from the rest, such as the one on the top of the page, but the others are not clear from the layout. Is the text surrounding the central photograph one single article or are there rather two articles? Does the first long column on the left continue in the small column below the photograph, or does one have to read next the long column on the right of the photograph? This can be determined only after examining the textual content of the page.

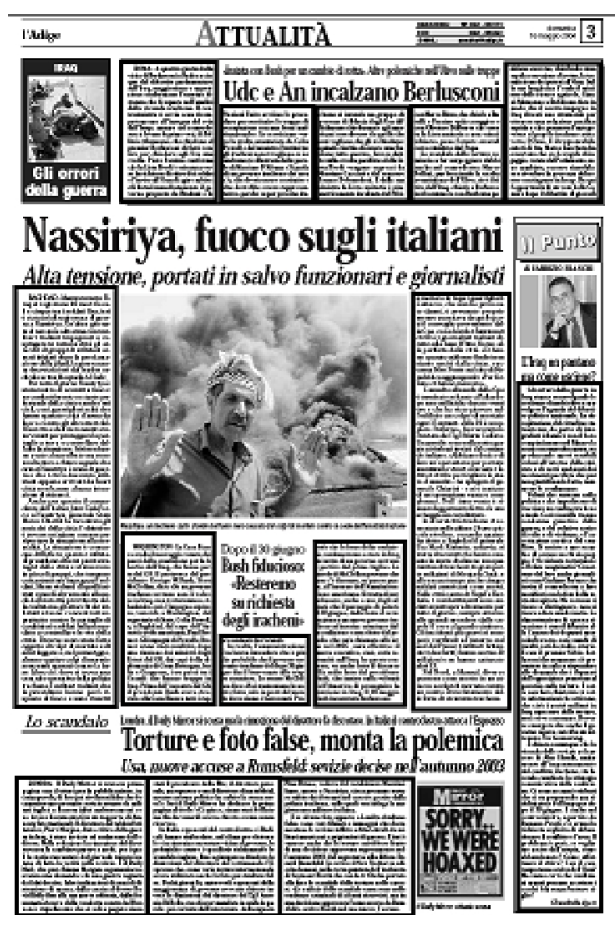

Figure 1: A page from L'Adige.

\subsection{Problem definition}

A document is composed of elementary logical units called document objects. Examples of document objects are the title, a figure, or a caption. Important characteristics of a document object are its bounding box, its position within the page, and its content. We focus on running text document objects which form the contents of an article. For the sake of simplicity, we ignore titles, subtitles and captions; we also ignore any spatial and syntactic information: the only information we consider is the text inside blocks. Given a newspaper page, we assume that all the blocks of text in which the layout divides the articles are given, together with their textual content. No other information is assumed.

Definition. (article clustering) Given a set of running text document objects from a 
newspaper page, we call article clustering the problem of partitioning the document objects into disjoint sets of articles, so that the elements of each set taken in appropriate order form a coherent piece of text.

In the following we present three algorithms to perform article clustering based on the textual content of the document objects. These three algorithms follow a general scheme and differ in the final step. First we provide the general algorithm description and then the three variants.

\subsection{General algorithm}

In order to group the blocks of text into a number of articles (clusters), we must solve two problems, namely:

- find the features which best describe the document objects in an article,

- find the features which best distinguish the document object in an article from the objects in the other articles.

The first set of features provides a quantification of intra-cluster similarity, while the second one provides a quantification of inter-cluster dissimilarity [Baeza-Yates and Ribeiro-Neto, 1999].

Each article (cluster) is described by the set of the words it contains (intra-cluster similarity) and is differentiated from the others by the presence of terms not present (or present in smaller number) in the other articles (inter-cluster dissimilarity).

A method to state whether two blocks belong to the same article is to compare the words they contain: if they share the same words (or groups of words, or synonyms) they are likely to be about the same subject. Not all the terms are equally useful for describing the document contents, and therefore term frequency must be considered. As usual in Information Retrieval, we assign a numerical weight to each term in a block; this value includes a quantification of both the intra-cluster similarity and the inter-cluster dissimilarity. The first one is represented by the frequency of the term in its block and provides a measure of how well it describes the subject in the block, while the second one is represented by a measure of the rarity of the term in the page. These two values are then combined into a single measure.

In this manner, each block is described by a list of terms and their corresponding weights. We can look at the block as $n$-dimensional vectors, one dimension for each word listed. In this way one represents the blocks in the page as a group of vectors in an $n$-dimensional space, and then considers the angle between two vectors as a measure of the similarity between them. When we know the degree of similarity between all the pairs of blocks, we can group together all the blocks which are "sufficiently near" and state that they belong to the same article. 
The general algorithm for article clustering consists of four steps:

1. Indexing: obtain the list of all the words inside the blocks,

2. Weighting: give a weight to each word inside each block,

3. Computing similarity: find the similarity between all the pairs of vectors,

4. Clustering: group together the blocks which probably belong to the same article.

Let us consider each step of the algorithm.

\subsubsection{Indexing}

With the process of indexing we reduce the text to a list of index terms or keywords; the set of all the index terms provides a logical view of the block. An index term is a text word whose semantics helps in identifying the document contents.

This logical view can be full text if it lists all the words in the text, or it can include only some of them, through the elimination of stopwords (functional words, such as articles, connectives, auxiliary verbs), the use of stemming (reduction of distinct words to their grammatical root), the reduction of synonyms to a single term and the grouping of sequences of words with a single lexical meaning (such as "forza multinazionale" - multinational force, or the first and last name of people). We do not keep any information about the position of terms in the text, but we keep track of the number of times each term appears, associating each keyword in the list with its number of occurrences.

For each page this step outputs a text file containing, for each block of text, a list of terms and their frequency in the block. However, the algorithms used to perform these operations are not a subject of this paper and we refer to [Tonella et al., 2004] for details.

\subsubsection{Weighting}

By parsing the index files, we obtain the list of words and their frequency in the blocks, in other words a frequency vector for each block. Starting from this information we build, for each block, a $t$-dimensional vector (where $t$ is the total number of distinct words in the index) where each element represents the weight of a particular word in the block. Next we define the weight vector.

Definition. (weight vector) Let $b$ be the number of blocks in the page, $t$ be the number of terms in the page, and $k_{i}$ be a generic index term. Let $P=\left\{b_{1}, b_{2}, \ldots, b_{b}\right\}$ be the complete set of text blocks in a page, and $K=\left\{k_{1}, k_{2}, \ldots, k_{t}\right\}$ be the set of all index terms. We associate 
a value $w_{i, j}$ called weight with each index term $k_{i}$ of a block $b_{j}$. A weight vector of a block $b_{j}$ is a sequence of weights $\left\{w_{1, j}, w_{2, j}, \ldots, w_{t, j}\right\}$. If a term $i$ does not appear in the $j$-th block, then we set $w_{i, j}$ to 0 .

The weight of a term $k_{i}$ of a block $b_{j}$ is computed in the standard way as the product of the normalized term frequency and the inverse document frequency [Sparck Jones, 1972]:

$$
w_{i, j}=t f_{i, j} \times i d f_{i}=\frac{f r e q_{i, j}}{\max _{k} f r e q_{i, j}} \times \log \frac{b}{n_{i}}
$$

where $n_{i}$ is the number of blocks in which the term $k_{i}$ appears.

\subsubsection{Computing the similarity}

The next step is to compute a matrix, called the similarity matrix, where the element $(h, k)$ represents the degree of similarity between the block $h$ and the block $k$. The similarity is represented by the cosine of the angle between the weight vectors related to the two blocks $b_{h}$ and $b_{k}$ [Salton and McGill, 1983, Baeza-Yates and Ribeiro-Neto, 1999]. Each element $(h, k)$ of the similarity matrix is given by the following $\operatorname{sim}(h, k)$ function:

$$
\operatorname{sim}(h, k)=\frac{b_{h} \bullet b_{k}}{\left|b_{h}\right| \times\left|b_{k}\right|}=\frac{\sum_{i=1}^{t} w_{i, h} \times w_{i, k}}{\sqrt{\sum_{i=1}^{t} w_{i, h}^{2}} \times \sqrt{\sum_{i=1}^{t} w_{i, k}^{2}}}
$$

The higher the similarity between two blocks, the higher the probability that they are in the same article.

\subsubsection{Clustering}

The clustering process consists of aggregating single blocks into sets of blocks (clusters). We represent the document objects as the nodes of a graph, called the connection graph. An edge represents the fact that two objects belong to the same cluster. In Figure 2, a connection graph is shown where three clusters are formed, that is, the cluster formed by $\left\{b_{1}, b_{3}, b_{4}, b_{7}\right\}$, the one formed by $\left\{b_{2}, b_{5}\right\}$ and the singleton $\left\{b_{6}\right\}$.

The three algorithms for performing the article clustering differ only in this last step. All the algorithms start with a graph with one node per document object and no edges. At each step one or more edges are added to the graph. At each iteration of the algorithm each connected sub-graph represents a portion of an article. The output of the algorithm is a graph in which each fully connected component represents a cluster, that is, a complete article. In all the three variants, we fix a similarity threshold: all pairs of blocks with similarity greater than the threshold can be considered part of the same article. 


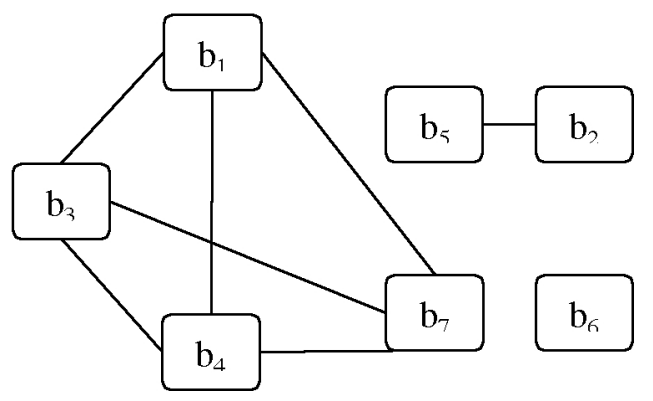

Figure 2: A connection graph.

\subsection{Simple Clustering Algorithm}

In the simple clustering algorithm (SCA) an edge in the connection graph is set by looking at the similarity matrix. For each element, if the value is above the threshold, then there is an edge in the graph. This algorithm is shown in Figure 3.

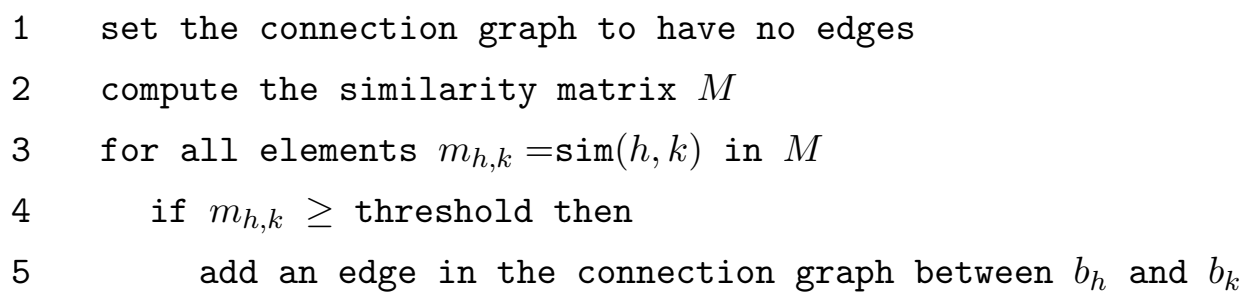

Figure 3: The Simple Clustering Algorithm (SCA).

\subsection{Comparative Clustering Algorithm}

In the simple clustering algorithm all the edges are added at once. On the contrary, in the comparative clustering algorithm (CCA) the process of adding an edge is iterative and considers the edges present at each step in the graph. Let $\operatorname{subgraph}(b)$ be the set of nodes which are connected to $b$ and $e_{b}$ a generic element of it. The CCA algorithm is shown in Figure 5. The CCA algorithm searches for the blocks which are more similar and then it compares the partially formed clusters of blocks before adding a new edge. In Figure 4, two nodes are considered to be joined by an edge only if all the nodes that they are connected to have an inter-similarity greater than zero. The rationale is that all the blocks in an article must have a minimum value of similarity between them greater than 0 . 


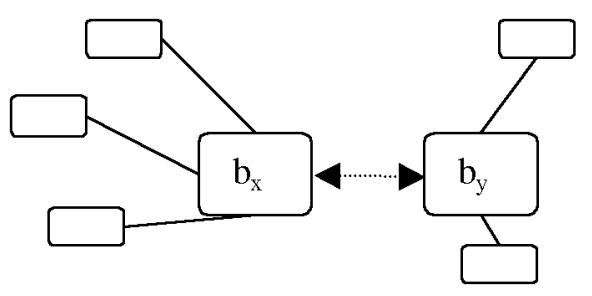

Figure 4: Comparing the subgraphs.

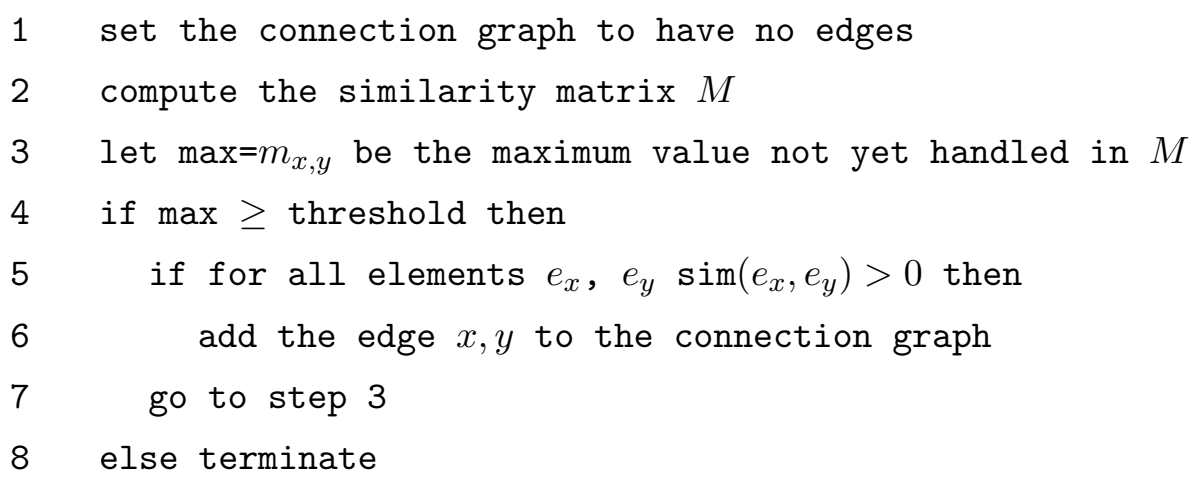

Figure 5: The Comparative Clustering Algorithm (CCA).

\subsection{Agglomerative Clustering Algorithm}

The idea of the Agglomerative Clustering Algorithm (ACA) is to extend the motivation behind the Comparative Clustering. When two very similar blocks are found, the two blocks are linked not only via an edge, but are merged into a single block. This implies that after the merge, all the weights of the blocks need to be recomputed. For each term appearing in at least one of the vectors of $x$ and $y$ the new frequency vector of the block $x y$ is given by the following values:

$$
\operatorname{freq}_{i, x y}= \begin{cases}\frac{\text { freq }_{i, x}+f r e q_{i, y}}{2} & \text { if } \text { freq }_{i, x} \neq 0 \text { and } \text { freq }_{i, y} \neq 0 \\ \operatorname{freq}_{i, x} & \text { if } f r e q_{i, x} \neq 0 \text { and } \text { freq }_{i, y}=0 \\ \text { freq }_{i, y} & \text { if } \text { freq }_{i, x}=0 \text { and } \text { freq }_{i, y} \neq 0 \\ 0 & \text { otherwise }\end{cases}
$$

The idea behind the algorithm is illustrated in Figure 7. At each step, the two blocks which are more similar and have a similarity above the threshold are merged into a unique block. In this way, the merged blocks have more power in "attracting" the other blocks of the same article. On the other hand, when two wrong blocks are merged they loose most of the 
attracting power towards the groups of the two different articles they belong two, leading to unpredictable results. The reason why we merge the two frequency vectors in such an unusual way lays on the necessity of maintaining the balance in the dimension of the blocks. After few steps of the algorithm there can be a block made of two, three or more blocks, with very high frequency values; experiments not reported in this document show that this unbalance worsens the algorithms performance.

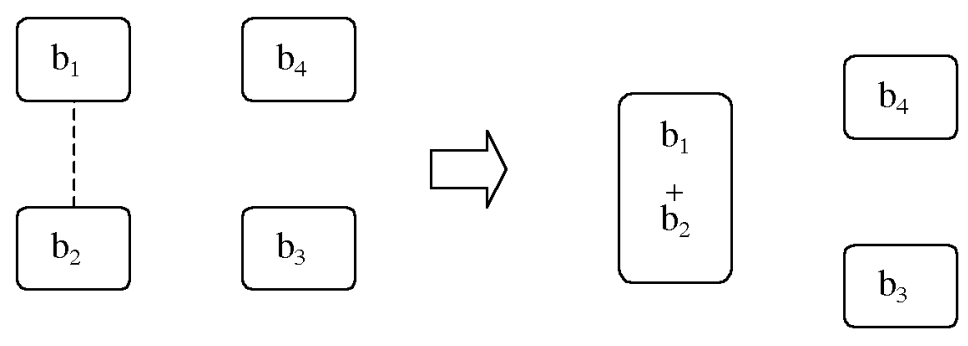

Figure 6: Merging similar blocks.

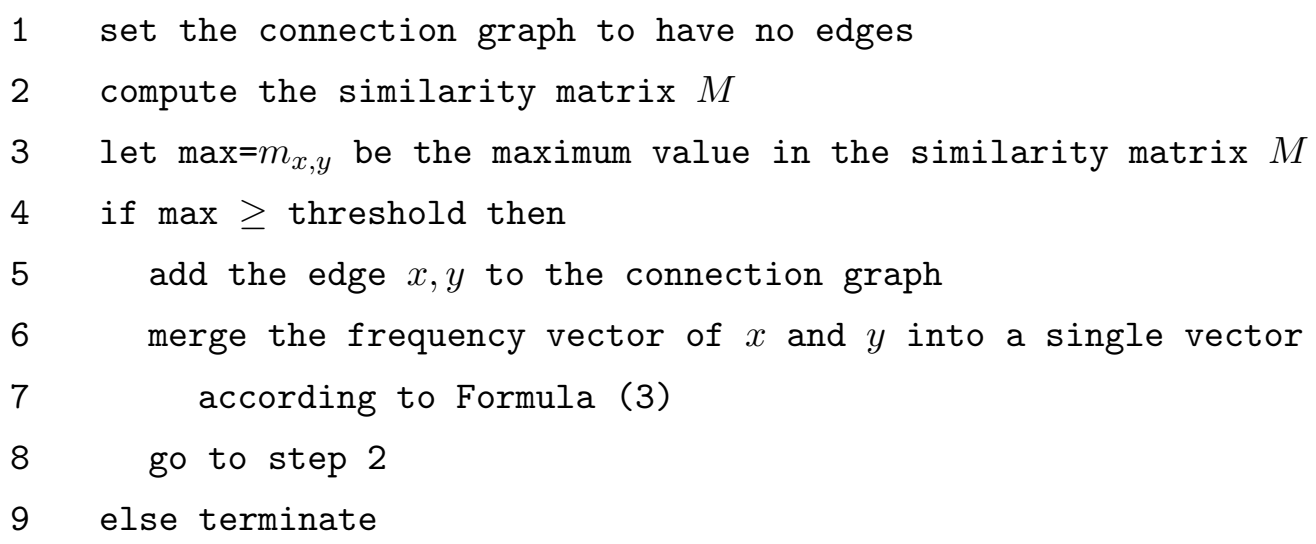

Figure 7: The Agglomerative Clustering Algorithm (ACA).

In summary, the Simple Clustering Algorithm has two main advantages: simplicity and efficiency. The Comparative Clustering adds only a little complexity compared to the Simple Clustering algorithm; the exclusion of some edges in the connection graph should entail an improvement in the correctness of the edges set, with a possible loss in the portion of edges found. Finally, the Agglomerative Clustering Algorithm may improve both correctness and completeness, with a significant handicap: it is slower than the other two, since it must re-compute several times all the weights and the similarity matrix. 


\section{$3 \quad$ Experimental results}

To test whether the proposed algorithms are effective to solve the article clustering problem and to discover which of the strategies is the best, we have run them on a real and meaningfully heterogeneous collection of newspaper pages from L'Adige (http://www.ladige.it). The algorithms work by comparing the similarity of two blocks with a given threshold. The value of this threshold is established by performing appropriate experiments.

The first step in the experiment is the setting of adequate accuracy measures that can give a quantitative value to the output of the algorithm. Given a newspaper page and the correct clustering of the document objects into articles as determined by a human being (ground truth), how close is the output of an article clustering algorithm? To answer this question we introduce the notions of precision, recall, and distribution.

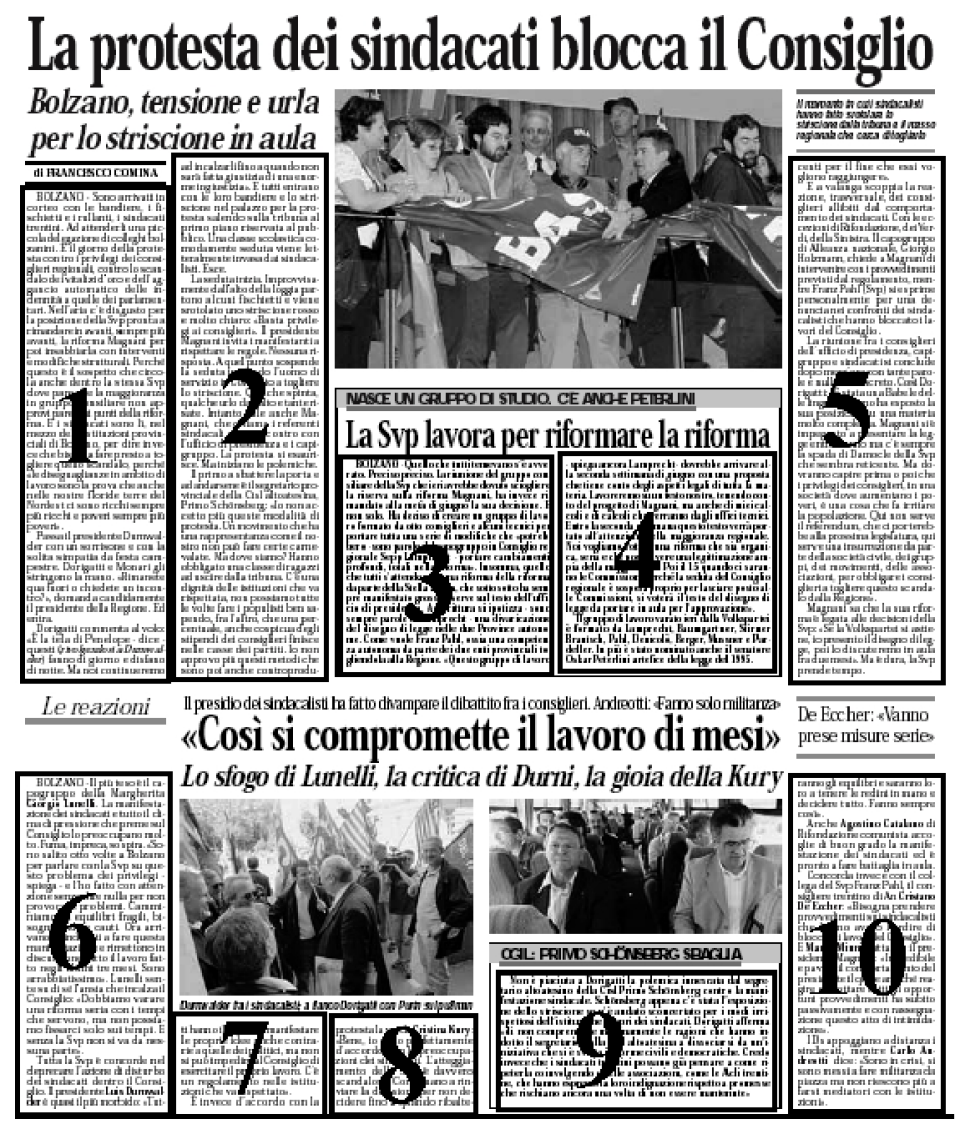

Figure 8: Identification of running text blocks in a page from L'Adige. 


\subsection{Measures}

Consider the page from L'Adige in Figure 8. The textual document objects are identified by integers ranging from 1 to 10, which form 4 articles. The first article, "La protesta dei sindacati blocca il Consiglio" is made up by blocks 1,2 , and 5 ; surrounded by blocks 2 and 5 we find the second article, "La Svp lavora per la riforma", which is made up by blocks 3 and 4. The third article is "Così si compromette il lavoro di mesi," composed by blocks 6, 7, 8, and 10; the last article "CGIL: Primo Schonsberg Sbaglia" is composed by block 9 alone. In this case the ground truth is (Figure 9):

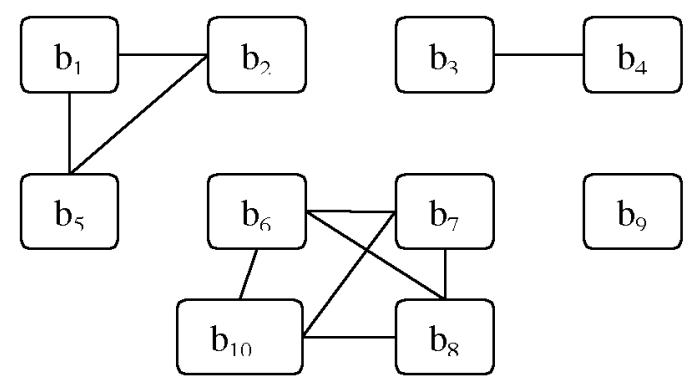

Figure 9: The ground truth for the article in Figure 8.

Article 1: block 1, block 2, block 5

Article 2: block 3, block 4

Article 3: block 6, block 7, block 8, block 10

Article 4: block 9

Assume next that the output of a clustering algorithm is the following (Figure 10):

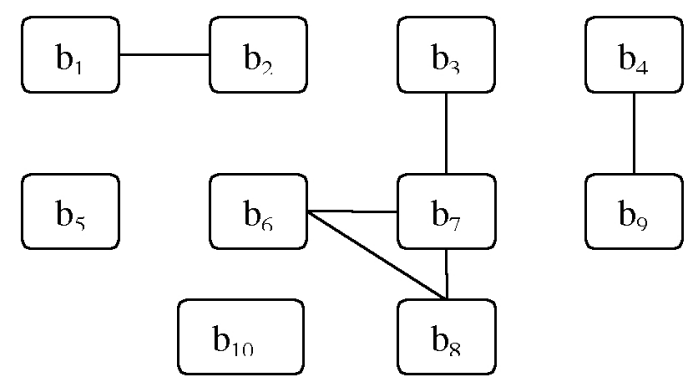

Figure 10: A clustering output for the article in Figure 8. 
Article 1: block 1, block 2

Article 2: block 3, block 6, block 7, block 8

Article 3: block 5

Article 4: block 4, block 9

Article 5: block 10

To measure the difference between the output and the golden truth, we compare the respective connection graphs. In Figure 11, we merge the two graphs, highlighting the correct edges present in the output graph, the missing and the wrongly added ones.
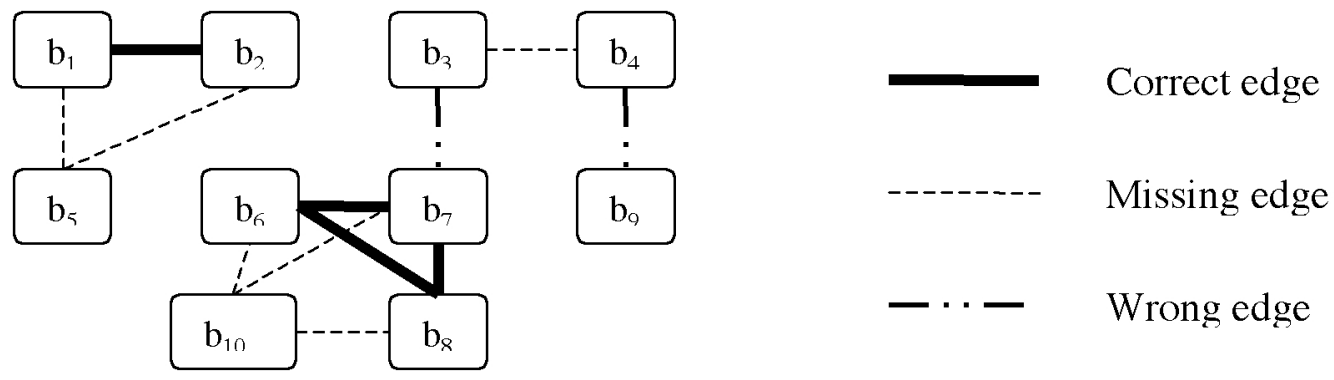

Figure 11: Comparing the connection graphs of the output and ground truth.

We use two measures taken from the information retrieval literature (see for instance [Baeza-Yates and Ribeiro-Neto, 1999]): recall represents the fraction of edges in the ground truth which are in the output, and precision represents the fraction of output edges which are in the ground truth graph. In addition, we use another measure to compare the number of articles retrieved which we call distribution, which represents the fraction between the number of clustered articles and the number of true articles or, to keep this number in the range 0-1, the vice-versa. More formally, if

Truth edges $=$ number of edges present in the truth graph

Output edges $=$ number of edges present in the output graph

Correct edges $=$ number of edges of the output graph that appear in the truth graph

Truth CC = number of connected components (i.e., articles) in the ground truth

Output $\mathrm{CC}=$ number of connected components found in the output

then we have 


$$
\begin{aligned}
\text { Precision } & =\frac{\text { Correct edges }}{\text { Output edges }} \\
\text { Recall } & =\frac{\text { Correct edges }}{\text { Truth edges }} \\
\text { Distribution } & = \begin{cases}\frac{\text { Truth CC }}{\text { Output CC }} & \text { if Truth CC } \leq \text { Output CC } \\
\frac{\text { Output CC }}{\text { Truth CC }} & \text { otherwise }\end{cases}
\end{aligned}
$$

If the algorithms cluster many wrong blocks there will be high recall values opposed to very low precision values; viceversa if the algorithms join just a small number of correct blocks, we have good precision values (even 100\%) with bad recall values. For example, if the algorithm joins all of the blocks in a single article, we have a recall value of $100 \%$ (there are edges between all the pairs of blocks, among which there are all the correct edges) coupled with a very bad precision value.

A source of a potential misreading of the results is that even with high values in precision and recall, we may be clustering wrongly the articles in the paper. For instance, many correct edges may have been identified, but a few wrong edges connect different articles, thus yielding many wrong clusters. Therefore, we need a measure that highlights the number of articles clustered compared to the number of actual articles in the page. The distribution captures the gap between actual articles and clustered ones.

Finally, another problem needs to be addressed. Suppose to have two outputs obtained with different thresholds. The first one brings a precision of $60 \%$, a recall of $40 \%$ and a distribution of $70 \%$. The other one has a precision of $50 \%$, a recall of $45 \%$ and a distribution of $75 \%$. Which of these is better? In order to answer this question we need a mean measure. Note that a standard measure for combining precision and recall such as the F-measure is not helpful here, as it does not take into account the clusters of articles, but only the edges in the graph. We define the Weighted Harmonic Mean (WHM) of precision, recall, and distribution as

$$
\mathrm{WHM}=\frac{5}{\frac{2}{\text { precision }}+\frac{2}{\text { recall }}+\frac{1}{\text { distribution }}}
$$

The harmonic mean is weighted for the purpose of giving less importance to the distribution value, which is useful but very imprecise. Since the distribution is merely a ratio between the numbers of articles, it can show good values even if precision and recall are very bad. 
One may want to reduce all the results to single values of precision, recall and distribution, applicable to the entire group of tests. There are two ways to combine the evaluations: a macro-mean or a micro-mean. Macro-mean is the normal mean value, obtained by adding the values and then dividing the result by the number of operands. Let $n$ be the number of executed tests, Correct edges ${ }_{i}$ be the number of correct edges found in the $i$-th test, Output edges $_{i}$ be the number of output edges found in the $i$-th test and so on; then the micro-mean is defined as follows:

$$
\begin{aligned}
& \text { Precision micro-mean }=\frac{\sum_{i=1}^{n}{\text { Correct } \text { edges }_{i}}^{n}}{\sum_{i=1}^{n} \text { Output edges }_{i}} \\
& \text { Recall micro-mean }=\frac{\sum_{i=1}^{n}{\text { Correct } \text { edges }_{i}}_{\sum_{i=1}^{n} \text { Truth edges }}}{\text { The }_{i}} \\
& \text { Distribution micro-mean }= \begin{cases}\frac{\sum_{i=1}^{n} \text { Truth } \mathrm{CC}_{i}}{\sum_{i=1}^{n} \text { Output } \mathrm{CC}_{i}} & \text { if } \sum_{i=1}^{n} \text { Truth } \mathrm{CC}_{i} \leq \sum_{i=1}^{n} \text { Output } \mathrm{CC}_{i} \\
\frac{\sum_{i=1}^{n} \text { Output } \mathrm{CC}_{i}}{\sum_{i=1}^{n} \text { Truth } \mathrm{CC}_{i}} & \text { otherwise }\end{cases}
\end{aligned}
$$

The WHM of the micro-mean is computed over the three micro-mean values.

\section{$3.2 \quad$ Experimental set up}

We used a document collection with 24 pages taken from different parts of the newspaper L'Adige. We chose this newspaper for its complex layout and the presence of many articles in each individual page; moreover, on the web-site http://www.ladige.it pdf and html reproduction of each page are published daily. Half of the pages in the collection were used in the determination of the similarity threshold, the other half of the pages were used in the determination of the performances of the algorithms. The algorithms and the evaluation procedure were implemented in Perl, and the experiments were run on a standard Pentium $42400 \mathrm{PC}$ with $256 \mathrm{MB}$ of RAM memory running the Windows XP operating system. The complete experimental results are presented in [Pegoretti, 2004].

The distribution of the articles and blocks in the pages is displayed in Figure 12. The second column refers to the pages used for the first two tests, while the third column refers to the final test. 


\begin{tabular}{|l|c|c|c|}
\hline & total & first 12 & last 12 \\
\hline \hline Articles & 161 & 72 & 89 \\
Blocks & 407 & 198 & 209 \\
Average articles per page & 6.7 & 6 & 7.4 \\
Average blocks per page & 17 & 16.5 & 17.4 \\
Average blocks per article & 2.5 & 2.8 & 2.3 \\
\hline
\end{tabular}

Figure 12: Article distribution in the experimental data set.

\subsubsection{Indexing}

To test the three algorithms independently of the indexing technique used in the first phase, we used three different indexing strategies.

Base indexing: One way to index is simply to report all the words in the text with their respective frequency. In addition we reduce plurals and singulars to a single word (like 'governo'/'governi government/governments) and a small number of groups of synonyms or expressions with the same meaning (e.g., 'prezzo'/'costo' 'price'/'cost' but also 'Washington'/'capitale Americana' 'Washington'/'American capital').

Stop indexing: The only advantage of the previous approach is processing speed. On the negative side, it leaves in the index all the articles, conjunctions, auxiliary verbs etc. These words are not only so common that one can find them in all the blocks in the page, but moreover they do not add any semantic information. Therefore the second step is to eliminate all these common words, called stopwords.

Bigram indexing: The third strategy uses elimination of the stopwords and the combination of pairs of words that make a single lexical unit (bigrams): 'dibattito parlamentare' (parliament discussion) or 'forza multinazionale' (multinational force).

In Figure 13 we summarize the distribution of terms and their occurrences in the blocks, as obtained from the test pages indexed in the three ways. We display information about the entire collections and separately about the two halves of the data set.

\subsection{Determination of the similarity threshold}

We use the Weighted Harmonic Mean to determine the optimal similarity threshold; that is, the threshold which yields the highest micro-mean WHM is the one to be chosen. The way to choose the threshold is to make tests on a set of documents representative of the collection for which the ground truth is available. 


\begin{tabular}{|l|c|c|c|}
\hline & base & stop & bigram \\
\hline \hline Over all the pages: & & & \\
Average number of terms per block & 90.0 & 53.9 & 50.8 \\
Average number of occurrences per term & 1.4 & 1.1 & 1.1 \\
Average number of terms per page & 933 & 747 & 731 \\
\hline First 12 pages: & & & \\
Average number of terms per block & 90.2 & 53.3 & 50.2 \\
Average number of occurrences per term & 1.4 & 1.1 & 1.1 \\
Average number of terms per page & 910 & 720 & 703 \\
\hline Last 12 pages: & & & \\
Average number of terms per block & 89.8 & 54.5 & 51.4 \\
Average number of occurrences per term & 1.4 & 1.1 & 1.1 \\
Average number of terms per page & 957 & 774 & 759 \\
\hline
\end{tabular}

Figure 13: Data set distribution with respect to indexing strategies.

Note that if the threshold is too low, the algorithms combine many unrelated blocks, resulting in a high recall and low precision; on the other hand, a threshold that is too high means that fewer blocks are joined, leading to very good values in precision, but very bad ones in recall. Moreover, the relation between the two parameters is not linear; and therefore we should find a good balance between the extremes.

The first experiment was run to determine the optimal value for the threshold. We applied the tests to the first half of the data set, employing all three indexing strategies. For each page, we repeated the algorithms with different thresholds, evaluated the results and kept the threshold leading to the best WHM value. In Figure 14, we summarize the results.

\begin{tabular}{|l|l|c|c|}
\hline best value & algorithm & macro-mean & micro-mean \\
\hline \hline WHM & Agglomerative clustering with stop indexing & $75.54 \%$ & $75.74 \%$ \\
\hline Precision & Comparative clustering with bigram indexing & $83.26 \%$ & $82.22 \%$ \\
\hline Recall & Agglomerative clustering with base indexing & $73.54 \%$ & $71.60 \%$ \\
\hline
\end{tabular}

Figure 14: Results for optimal value determination.

The second group of tests was devoted to the research of the similarity threshold that should be used by a program that implements the algorithms, i.e., a single threshold that should be applied to all the pages of a given set of documents. This threshold was determined on 
the same data set of the previous tests. Testing values around the mean value of the best thresholds found (in the latter test), we searched for the value that, when applied to all the first half of the pages in the collection, yielded the best macro and micro mean of the performance evaluations. We tested all the three algorithms on all the three groups of vectors, as done before. In Figure 15, we summarize the results.

\begin{tabular}{|l|l|c|c|}
\hline best value & algorithm & macro-mean & micro-mean \\
\hline \hline WHM & Agglomerative clustering with bigram indexing & $70.95 \%$ & $71.50 \%$ \\
\hline Precision & Comparative clustering with bigram indexing & $77.36 \%$ & $73.26 \%$ \\
\hline Recall & Agglomerative clustering with bigram indexing & $72.60 \%$ & $68.80 \%$ \\
\hline
\end{tabular}

Figure 15: Results for the threshold value determination.

Finally, we tested the algorithms working with a threshold chosen a priori. We applied the fixed threshold found in the latter test to the second half of the document collection, and tested all the three algorithms over all the documents indexed in the three different ways. In Figure 17 we present the results with the base indexing strategy, in Figure 18 with the stop indexing strategy, in Figure 19 with the bigram indexing strategy, and in Figure 16, we summarize the overall experimental results, highlighting the best algorithms.

\begin{tabular}{|l|l|c|c|}
\hline best value & algorithm & macro-mean & micro-mean \\
\hline \hline \multirow{2}{*}{ WHM } & Simple clustering with stop indexing & $59.77 \%$ & $58.91 \%$ \\
& Comparative clustering with bigram indexing & $59.72 \%$ & $58.25 \%$ \\
\hline Precision & Simple clustering with stop indexing & $65.41 \%$ & $58.25 \%$ \\
\hline Recall & Agglomerative clustering with stop indexing & $66.90 \%$ & $67.02 \%$ \\
\hline
\end{tabular}

Figure 16: Results for the threshold value determination.

\subsection{Discussion}

The three algorithms give very similar results. The best results for each test (obtained with the stop indexing and the bigram indexing) differ in a few percentage points. In the Best Result tests, where Agglomerative Clustering with bigram indexing obtained a WHM value of $75.74 \%$ (micro-mean), the other two algorithms proved to be almost equally good: Simple Clustering obtained with stop indexing a WHM of $73.34 \%$ and Comparative Clustering a WHM of $73.23 \%$. In the other tests the situation does not vary much: the best results of each algorithm never differ by more than $3 \%$. There is apparently no need for the additional 


\begin{tabular}{|c|c|c|c|c|c|}
\hline \multicolumn{6}{|c|}{ Simple clustering with base indexing } \\
\hline & threshold & WHM & precision & recall & distribution \\
\hline Best & 0.0802 & $87.5 \%$ & $91.7 \%$ & $84.6 \%$ & $85.7 \%$ \\
\hline Worst & 0.0802 & $18.3 \%$ & $33.3 \%$ & $10.0 \%$ & $75.0 \%$ \\
\hline Macro-mean & 0.0802 & $48.4 \%$ & $55.6 \%$ & $53.5 \%$ & $76.5 \%$ \\
\hline Micro-mean & & $47.3 \%$ & $36.6 \%$ & $51.3 \%$ & $83.2 \%$ \\
\hline \multicolumn{6}{|c|}{ Comparative clustering with base indexing } \\
\hline & threshold & WHM & precision & recall & distribution \\
\hline Best & 0.0802 & $87.5 \%$ & $91.7 \%$ & $84.6 \%$ & $85.7 \%$ \\
\hline Worst & 0.0802 & $18.3 \%$ & $33.3 \%$ & $10.0 \%$ & $75.0 \%$ \\
\hline Macro-mean & 0.0802 & $48.4 \%$ & $55.6 \%$ & $53.5 \%$ & $76.5 \%$ \\
\hline Micro-mean & & $47.3 \%$ & $36.6 \%$ & $51.3 \%$ & $83.2 \%$ \\
\hline \multicolumn{6}{|c|}{ Agglomerative clustering with base indexing } \\
\hline & threshold & WHM & precision & recall & distribution \\
\hline Best & 0.0704 & $87.5 \%$ & $91.7 \%$ & $84.6 \%$ & $85.7 \%$ \\
\hline Worst & 0.0704 & $86.0 \%$ & $37.0 \%$ & $71.4 \%$ & $80.0 \%$ \\
\hline Macro-mean & 0.0704 & $43.8 \%$ & $47.1 \%$ & $53.9 \%$ & $73.2 \%$ \\
\hline Micro-mean & & $39.5 \%$ & $25.8 \%$ & $52.4 \%$ & $89.9 \%$ \\
\hline
\end{tabular}

Figure 17: The experimental results using the base indexing strategy.

operations done by the two variants of the Simple Clustering Algorithm.

We can see how the Simple Clustering algorithm and the Comparative Clustering one give the same results when the base indexing is used. The reason for this behavior is that, with all stopwords included in the vectors, each block always has a similarity degree with all the other blocks different from 0 (since they always share some terms), so that the Comparing Clustering does not prevent any edge of the connection graph from being added. The Agglomerative Clustering always shows the worst performances when it uses vectors indexed with base indexing. This way of indexing increases the probability of making errors; when the algorithm merges two wrong blocks the errors tend to propagate. Compared to the Simple Clustering, we can state that it is more robust but less stable: it commits less errors, but when it incurs in an error the results are unpredictable.

The best and the worst results always correspond to the same documents. The worst 


\begin{tabular}{|c|c|c|c|c|c|}
\hline \multicolumn{6}{|c|}{ Simple clustering with stop indexing } \\
\hline & threshold & WHM & precision & recall & distribution \\
\hline Best & 0.0572 & $89.6 \%$ & $100 \%$ & $80.0 \%$ & $92.3 \%$ \\
\hline Worst & 0.0572 & $00.0 \%$ & $00.0 \%$ & $0.0 \%$ & $80.0 \%$ \\
\hline Macro-mean & 0.0572 & $59.8 \%$ & $65.4 \%$ & $59.4 \%$ & $82.3 \%$ \\
\hline Micro-mean & & $58.9 \%$ & $51.4 \%$ & $59.2 \%$ & $82.4 \%$ \\
\hline \multicolumn{6}{|c|}{ Comparative clustering with stop indexing } \\
\hline & threshold & WHM & precision & recall & distribution \\
\hline Best & 0.0440 & $89.6 \%$ & $100 \%$ & $80.0 \%$ & $92.3 \%$ \\
\hline Worst & 0.0440 & $18.3 \%$ & $33.3 \%$ & $10.0 \%$ & $75.0 \%$ \\
\hline Macro-mean & 0.0440 & $59.0 \%$ & $61.8 \%$ & $61.1 \%$ & $83.0 \%$ \\
\hline Micro-mean & & $58.3 \%$ & $50.2 \%$ & $57.6 \%$ & $89.0 \%$ \\
\hline \multicolumn{6}{|c|}{ Agglomerative clustering with stop indexing } \\
\hline & threshold & WHM & precision & recall & distribution \\
\hline Best & 0.0384 & $90.9 \%$ & $85.7 \%$ & $92.3 \%$ & $100 \%$ \\
\hline Worst & 0.0384 & $18.3 \%$ & $33.3 \%$ & $10.0 \%$ & $75.0 \%$ \\
\hline Macro-mean & 0.0384 & $57.0 \%$ & $54.5 \%$ & $66.9 \%$ & $87.3 \%$ \\
\hline Micro-mean & & $56.0 \%$ & $40.9 \%$ & $67.0 \%$ & $94.7 \%$ \\
\hline
\end{tabular}

Figure 18: The experimental results using the stop indexing strategy.

results are given by the pages with a low number of articles all discussing more or less the same subject, divided into many blocks. On the other hand, the best results are given where few articles discuss heterogeneous topics and are divided into a small number of big blocks. Even a human reader, given the raw text of the blocks, can have serious difficulties in the most complex cases in reconstructing the different articles.

As expected, the base indexing proved to be the worst type of indexing, leading to the worst results in every test. In particular, in the Best Result tests, it leads to a great number of wrong edges, bringing high recall value and very low precision values. Stop indexing and bigram indexing gave more or less the same results: sometimes the best result is obtained with stop indexing, sometimes with bigram indexing. Since the first one is simpler, it is preferable to the second one, but the data set is too small to exclude one of the two. Actually, bigram indexing can give good results in most cases, since the Keyword-extractor system we used to 


\begin{tabular}{|c|c|c|c|c|c|}
\hline \multicolumn{6}{|c|}{ Simple clustering with bigram indexing } \\
\hline & threshold & WHM & precision & recall & distribution \\
\hline Best & 0.0446 & $89.6 \%$ & $100 \%$ & $80.0 \%$ & $92.3 \%$ \\
\hline Worst & 0.0446 & $20.7 \%$ & $09.6 \%$ & $100 \%$ & $80.0 \%$ \\
\hline Macro-mean & 0.0446 & $57.0 \%$ & $61.9 \%$ & $63.7 \%$ & $85.4 \%$ \\
\hline Micro-mean & & $55.4 \%$ & $42.3 \%$ & $62.8 \%$ & $89.9 \%$ \\
\hline \multicolumn{6}{|c|}{ Comparative clustering with bigram indexing } \\
\hline & threshold & WHM & precision & recall & distribution \\
\hline Best & 0.0384 & $89.6 \%$ & $100 \%$ & $80.0 \%$ & $92.3 \%$ \\
\hline Worst & 0.0384 & $24.5 \%$ & $11.5 \%$ & $100 \%$ & $100 \%$ \\
\hline Macro-mean & 0.0384 & $59.7 \%$ & $62.9 \%$ & $60.6 \%$ & $79.2 \%$ \\
\hline Micro-mean & & $58.3 \%$ & $51.4 \%$ & $56.5 \%$ & $86.4 \%$ \\
\hline \multicolumn{6}{|c|}{ Agglomerative clustering with bigram indexing } \\
\hline & threshold & WHM & precision & recall & distribution \\
\hline Best & 0.0291 & $81.1 \%$ & $83.3 \%$ & $76.9 \%$ & $85.7 \%$ \\
\hline Worst & 0.0291 & $20.7 \%$ & $09.6 \%$ & $100 \%$ & $80.0 \%$ \\
\hline Macro-mean & 0.0291 & $56.4 \%$ & $55.4 \%$ & $66.1 \%$ & $84.4 \%$ \\
\hline Micro-mean & & $54.5 \%$ & $39.7 \%$ & $64.4 \%$ & $96.7 \%$ \\
\hline
\end{tabular}

Figure 19: The experimental results using the bigram indexing strategy.

compute the indexes is still imprecise and the errors eliminate the advantages brought by the right bigrams found.

The best algorithm proved to be Agglomerative Clustering applied to vectors given by stop indexing, but only by less than $2 \%$. This is due to the fact that, even if it has the same best results as Simple Clustering, it has only 2 pages with a WHM value under $60 \%$ compared to 4 pages for Simple Clustering. Since Agglomerative Clustering with base indexing gives the best recall value coupled with the worst precision value, we see that when the algorithm makes a mistake it tends to put together wrong blocks and not to separate similar blocks. The Comparative Clustering algorithm proved to be the most precise among the three, with a consequent loss in recall, due to the prevention of both wrong and right edges. Some articles include blocks that have a degree of similarity of 0 with other blocks of the same article, and the these are avoided by the algorithm. However, since these are quite rare the worsening 
is roughly equivalent to the improvement (i.e., the number of deleted correct edges roughly equals the number of missed wrong edges).

As for the determination of the threshold, one sees the (relative) weakness of the Simple Clustering opposed to the robustness of the other two algorithms, which have the best results in WHM, precision and recall. Our conclusion is that the Agglomerative Clustering, if applied with a good threshold, can give the best overall results. The Comparative Clustering produces the best results in precision, due to the fact that it checks all the edges before adding them to the graph.

In the overall experiments, we conclude that the Simple Clustering algorithm is the one with overall best performance, proving to be not only the simplest and to have less computational complexity, but also to be more flexible than the other two.

\section{Complexity analysis}

It is interesting to compare the three algorithms not only from the point of view of their performance, as we did in the previous section, but also in terms of their complexity. In the following we consider worst case time complexity, best case and present a representative case. We show that the three algorithms are all polynomial, but have increasing time costs.

We assume that we have $b$ blocks per page and that the total number of distinct words (per page) is $n$. To compute the number of operations to perform in a typical case, ${ }^{1}$ we consider the following numbers:

$\begin{array}{lrl}\text { different words per page } & n=1000 \\ \text { different words per block } & n_{b}=100 \\ \text { number of blocks } & b=21 \\ \text { number of articles } & a=7\end{array}$

\subsection{Indexing, Weighting and Similarity Matrix computation complexity}

In indexing, when we parse the lists of the indexed terms, we obtain $b$ vectors with the frequency of the terms. Each vector has only a number of elements equal to the number of different words in the single block, which is much less than $n$; we have therefore no need to allocate space for the missing terms, since we already know that their frequency is 0 . In the worst case each block has $n$ different words, whereas in the best case each word appears just in one block and the number of different words in a block is $\frac{n}{b}$. Usually, each block has approximately $\frac{n}{10}$ different terms with base indexing, and the ratio is even smaller with

\footnotetext{
${ }^{1}$ We talk of typical case, to avoid confusion with the well-known average complexity concept.
} 
stopword and bigram indexing. The exact complexity of the indexing is out of the scope of the current paper.

In weighting, one computes the weight of terms recurring in a given block, as the other terms have a weight of 0 . The weight is computed according to Equation (1). From the indexing step we already know $f r e q_{i, j}$ for each $i, j$ as well the number of blocks $b$, but we still have to compute the maximum frequency present in each block, and the number $n_{i}$ of blocks in which the term $k_{i}$ appears. Both operations can be performed during the same loop by considering all the frequency vectors, which leads to a worst case complexity of $\mathrm{O}(b \times n)$. If each block has $\frac{n}{b}$ elements, we have $\mathrm{O}\left(\frac{n}{b} \times b\right)=\mathrm{O}(n)$ operations. In the typical case, the number of operations is: $100 \times 21=2100$ operations. As for the $i d f s$ values for all the terms, these are constant and can be computed in $\mathrm{O}(n)$. For the typical case, assume that logarithms and divisions require 3 operations, then we have a total of $3 \times n$ operations $=3000$ operations. Finally, the computation of the weights is simply a division and a product, that is, $\mathrm{O}(n \times b)$ in the worst case, $\mathrm{O}(n)$ in the best case and $2100 \times 2=4200$ operations in the typical case. In summary, for weighting we have

$$
\begin{array}{lrl}
\mathrm{O}(b \times n)+\mathrm{O}(n)+\mathrm{O}(b \times n)= & \mathrm{O}(b \times n) & \text { worst case } \\
\mathrm{O}(n)+\mathrm{O}(n)+\mathrm{O}(n)= & \mathrm{O}(n) & \text { best case } \\
2100+3000+4200= & 9300 \text { op } & \text { typical case }
\end{array}
$$

In computing the similarity matrix, one computes the similarity between all the pairs of blocks. Note that the similarity matrix is symmetric, and therefore the number of computations is only half the size of the matrix. The formula to compute the similarity is Equation (2). In both the worst and best cases, each vector contains all $n$ words of the article, yielding a complexity of $\mathrm{O}(n)$. In the typical case, some terms will be missing in both blocks. The experiments showed that one usually compares blocks with no more than 10 common terms. The norms of the vectors are constant through all the operations, so we can calculate them just once for each vector. These can be computed once by performing $n$ products plus a sum and a division, yielding the complexity of $\mathrm{O}(n)$ for the worst case, $\mathrm{O}\left(\frac{n}{b}\right)$ for the best case, and $10+1+1=12$ operation for the typical case. As for the computation of the norms, in the worst case the vector has $n$ elements, thus, we have to compute $n$ squares, $n$ sums, and a square root for a total of $\mathrm{O}(b n)$ operations. In the best case, each vector has $\frac{n}{b}$ elements so the we have $\mathrm{O}\left(\frac{n}{b} \times b\right)=\mathrm{O}(n)$. In the typical case, we have 100 elements, thus, 201 operations per block. In summary, for weighting we have 


$$
\begin{array}{lll}
\mathrm{O}\left(\frac{b(b-1)}{2} \times n\right)+\mathrm{O}(n \times b)= & \mathrm{O}\left(b^{2} \times n\right) & \text { worst case } \\
\mathrm{O}\left(\frac{b(b-1)}{2} \times \frac{n}{b}\right)+\mathrm{O}\left(\frac{n}{b} \times b\right)== & \mathrm{O}(b \times n) & \text { best case } \\
\frac{21 \times 20}{2} \times 21+201 \times 21= & 6741 \text { op } & \text { typical case }
\end{array}
$$

\subsection{Simple clustering complexity}

To compute the connection graph one needs to compare each element of the similarity matrix with the threshold value. Since the number of elements in the similarity matrix is $\frac{b(b-1)}{2}$, the cost is $\mathrm{O}\left(b^{2}\right)$. In Figure 20, the total complexity of the simple clustering algorithm is shown.

\begin{tabular}{|l|c|c|r|}
\hline \multicolumn{4}{|c|}{ Simple clustering complexity } \\
\hline phase & Worst Case & Best Case & Typical Case \\
\hline \hline Weighting & $\mathrm{O}(b \times n)$ & $\mathrm{O}(n)$ & 9300 \\
\hline Similarity matrix & $\mathrm{O}\left(b^{2} \times n\right)$ & $\mathrm{O}(b \times n)$ & 6741 \\
\hline Connection graph & $\mathrm{O}\left(b^{2}\right)$ & $\mathrm{O}\left(b^{2}\right)$ & 289 \\
\hline \hline Total & $\mathrm{O}\left(b^{2} \times n\right)$ & $\mathrm{O}\left(b^{2}+b \times n\right)$ & 16300 \\
\hline
\end{tabular}

Figure 20: Simple clustering complexity.

\subsection{Comparative Clustering complexity}

In comparative clustering, the computation of the connection graphs is more expensive, as several cycles are necessary. In the worst case, all the blocks make up a single article and each block has $n$ distinct words.

To connect all $b$ blocks we have to discover $b-1$ edges, so that we must use the first $b-1$ best values in the similarity matrix. Suppose that we set in each iteration the maximum value to an infinite value, so each time we have to check all the $\frac{b(b-1)}{2}$ terms to find the maximum. In total $\frac{b(b-1)}{2} \times b(b-1)$ operations are required. If we sort the values in the matrix (e.g., in time $\mathrm{O}\left(\frac{b(b-1)}{2} \times \log \frac{b(b-1)}{2}\right)$ with a quicksort) this operation has no cost. It is easy to prove by induction that at the end of the process, independently of the order in which the connections have been discovered, we have to check $\frac{b(b-1)}{2} \times \frac{b(b-2)}{2}$ similarities between 
blocks, due to the checks made by the Comparison Clustering. In summary, the total cost with or without a quicksort respectively is:

$$
\begin{aligned}
\mathrm{O}\left(\frac{b(b-1)}{2} \times(b-1)+\frac{(b-1)(b-2)}{2}\right) & =\mathrm{O}\left(b^{3}\right) \\
\mathrm{O}\left(\frac{b(b-1)}{2} \times \log \frac{b(b-1)}{2}+\frac{(b-1)(b-2)}{2}\right) & =\mathrm{O}\left(b^{2} \times \log \left(b^{2}\right)\right)
\end{aligned}
$$

In the best case, we have no connection between the blocks and $\frac{n}{b}$ elements in each vector, and the cost is simply that of finding the best value in the similarity matrix. If we use quicksort, this is compensated by the fact that we know at no cost whether or not the maximum value is smaller than the threshold or not. In summary, the total cost respectively with or without a quicksort is:

$$
\begin{aligned}
\mathrm{O}\left(\frac{b(b-1)}{2}\right) & =\mathrm{O}\left(b^{2}\right) \\
\mathrm{O}\left(\frac{b(b-1)}{2} \times \log \frac{b(b-1)}{2}\right) & =\mathrm{O}\left(b^{2} \times \log \left(b^{2}\right)\right)
\end{aligned}
$$

In the typical case, with 21 blocks in 7 articles and 3 blocks per article, referring to the CCA algorithm on page 9 , we have:

$\begin{array}{ll}\text { step } 1 & 0 \text { operations } \\ \text { step } 2 & \frac{b(b-1)}{2}=210 \text { operations } \\ \text { step } 3 & 210 \text { operations } \\ \text { step } 4 & 1 \text { operation } \\ \text { step } 5 & 1 \text { operation } \\ \text { step } 6 & 1 \text { operation } \\ \text { step } 7 & 0 \text { operations } \\ \text { step } 8 & 0 \text { operations }\end{array}$

Step 3 to 6 are repeated for the 7 articles, plus the initialization operation. In total, we have 2961 operations. Note that, using a sorting algorithm, one would have 3038 operations, so in the typical case there is no practical advantage to use sorting. In Figure 21, the total complexity of the comparative clustering algorithm is reported.

\subsection{Agglomerative clustering complexity}

In the agglomerative clustering algorithm, the weights and the similarity matrix are computed several times during one run. We do not give here all the details of computing the complexity of each step of the algorithm, but only summarize the results: 


\begin{tabular}{|l|c|c|r|}
\hline \multicolumn{4}{|c|}{ Comparative clustering complexity } \\
\hline phase & Worst Case & Best Case & Typical Case \\
\hline \hline Weighting & $\mathrm{O}(b \times n)$ & $\mathrm{O}(n)$ & 9300 \\
\hline Similarity matrix & $\mathrm{O}\left(b^{2} \times n\right)$ & $\mathrm{O}(b \times n)$ & 6741 \\
\hline Connection graph & $\mathrm{O}\left(b^{3}\right)$ & $\mathrm{O}\left(b^{2}\right)$ & 2961 \\
\hline \hline Total & $\mathrm{O}\left(b^{2} \times n+b^{3}\right)$ & $\mathrm{O}\left(b^{2}+b \times n\right)$ & 19009 \\
\hline
\end{tabular}

Figure 21: Comparative clustering complexity.

\begin{tabular}{lc} 
Step & Total number of operations \\
\hline \hline Read frequency vector & $n$ \\
Compute $i d f s$ & $2 \times n$ \\
Compute weights & $2 \times n$ \\
Compute sim. matrix & $2(b-1)^{3}-6\left(b(b-1)^{2}\right)-(b-1)^{2}+6 b^{2}(b-1)-2(b-1)$ \\
\cline { 2 - 2 } Best value search & $2(b-1)^{3}-6\left(b(b-1)^{2}\right)-(b-1)^{2}+6 b^{2}(b-1)-2(b-1)$ \\
\cline { 2 - 2 } Vector merge & 12 \\
& $n$
\end{tabular}

For the worst case computing the weights has a total cost of $\mathrm{O}\left(b^{2} \times n\right)$, the cost for the similarity matrix is $\mathrm{O}\left(b^{3} \times n+b^{2} \times n^{2}\right), \mathrm{O}\left(b^{3}\right)$ for best value search and $\mathrm{O}(b \times n)$ for merging vectors. For the best case, the situation is analogous, except that the vectors have size $\frac{n}{b}$, which yields values of $\mathrm{O}(n), \mathrm{O}(b \times b), \mathrm{O}\left(b^{2}\right)$ and 0 for weighting, computing the similarity, searching for the best value and vector merging, respectively. Finally, for the typical case we have the following situation:

\begin{tabular}{lc} 
Step & Total number of operations \\
\hline \hline Read frequency vector & 29.400 \\
Compute $i d f s$ & 30.000 \\
Compute weights & 58.800 \\
Compute similarity matrix & 47.235 \\
Best value search & 1486 \\
Vector merge & 140
\end{tabular}

Summarizing, the worst and best case complexity of the agglomerative clustering algorithm are reported in Figure 22, together with the typical case. 


\begin{tabular}{|l|c|c|r|}
\hline \multicolumn{4}{|c|}{ Agglomerative clustering complexity } \\
\hline phase & Worst Case & Best Case & Typical Case \\
\hline \hline Weighting & $\mathrm{O}\left(b^{2} \times n\right)$ & $\mathrm{O}(n)$ & 118200 \\
\hline Similarity matrix & $\mathrm{O}\left(b^{3} \times n\right)$ & $\mathrm{O}(b \times n)$ & 47235 \\
\hline Best value search & $\mathrm{O}\left(b^{3}\right)$ & $\mathrm{O}\left(b^{2}\right)$ & 1486 \\
\hline Merging & $\mathrm{O}(b \times n)$ & 0 & 140 \\
\hline \hline Total & $\mathrm{O}\left(b^{3} \times n\right)$ & $\mathrm{O}\left(b^{2}+b \times n\right)$ & 167061 \\
\hline
\end{tabular}

Figure 22: Agglomerative clustering complexity.

\subsection{Discussion}

The three algorithms all have polynomial complexity, though there is a strict increase in worst case complexity, going from the simple algorithm to the comparative clustering one, and finishing with the agglomerative clustering algorithm. In Figure 23, we summarize the complexity of the three algorithms. One also notes that the best case cost is the same for all the three, and in particular, it is the cost of the computation of a single similarity matrix.

\begin{tabular}{|l|c|c|r|}
\hline \multicolumn{4}{|c|}{ Algorithms' complexity } \\
\hline Algorithm & Worst Case & Best Case & Typical Case \\
\hline \hline Simple Clustering & $\mathrm{O}\left(b^{2} \times n\right)$ & $\mathrm{O}\left(b^{2}+b \times n\right)$ & 16300 \\
\hline Comparative Clustering & $\mathrm{O}\left(b^{3}+b^{2} \times n\right)$ & $\mathrm{O}\left(b^{2}+b \times n\right)$ & 19002 \\
\hline Agglomerative Clustering & $\mathrm{O}\left(b^{3} \times n\right)$ & $\mathrm{O}\left(b^{2}+b \times n\right)$ & 167061 \\
\hline
\end{tabular}

Figure 23: Summary of the time complexity results.

The typical case gives us an indication that simple clustering and comparative have similar costs, on the other hand, agglomerative clustering is typically very expensive. The Agglomerative Clustering cost is one order of magnitude higher than the others, due to the iteration of the weighting step and of the computation of the similarity matrix.

\subsection{A note on execution times}

The execution times on the pages of the data-set confirm the theoretical complexity results of the previous section. In Figure 24, we show the execution times in milliseconds of the three algorithms using the three different indexing strategies implemented in Perl on a standard PC equipped with Pentium 42400 with 256 MB of RAM memory, running the Windows $\mathrm{XP}$ operating system. The times represent the average wall-clock time of the execution of 
the algorithms on all the data-set excluding the pre-indexing step which is the same for all algorithms.

\begin{tabular}{|l|r|r|r|}
\hline \multicolumn{4}{|c|}{ Algorithms' execution times } \\
\hline indexing strategy & base & stopword & bigram \\
\hline Clustering Algorithm & & & \\
\hline \hline simple (SCA) & 70 & 48 & 43 \\
comparative (CCA) & 72 & 49 & 45 \\
agglomerative (ACA) & 444 & 291 & 286 \\
\hline
\end{tabular}

Figure 24: Execution times in milliseconds for the three algorithms.

One notes that the simple and comparative clustering are very close in execution times and that the agglomerative one is significantly slower. Using the base indexing slows down computations as there are more words to compare, i.e., the vectors are bigger. The stopword and bigram strategies are similar with respect to execution time.

In summary, we conclude that the execution times are low, all below one second, and this makes the approach feasible to use as part of a document understanding systems.

\section{Conclusions}

Newspapers are a hard test for document understanding systems as they usually have a high number of document elements, several independent articles are scattered on the same page and have layouts which are not standardized and not geometrically simple.

We introduced three algorithms to address the article clustering problem within real newspaper pages. The three algorithms are based on text processing techniques and differ in their last step, that is, in the way the aggregation of blocks into articles is performed. The experiments, though performed on a small collection of pages, has shown how the performance of the algorithms does not differ much. In particular, the best results for each test (obtained sometimes with the stop indexing and sometimes with the bigram indexing) differ from another by a few percentage points. For instance, in the Best Result tests Agglomerative Clustering with bigram indexing obtained a WHM value of $75.74 \%$, while Simple Clustering obtained with stop indexing a WHM of $73.34 \%$ and Comparative Clustering with stop indexing obtained a WHM of $73.23 \%$. If the performance of the three algorithms is not so different, the difference in complexity is. All the algorithms are polynomial, but the worst case complexity is one degree less for the simple clustering algorithm.

The absolute values of the performance of the algorithms are in the range of $70 \%$. These 
values, which may appear to be good, but not outstanding, should be considered in the context of a whole document analysis architecture, where the proposed algorithms are a component of a larger system. Just as in [Aiello et al., 2002] the text processing component worked together with a spatial reasoner to identify unique reading orders from documents, a system for newspaper page understanding would have a document clustering component based both on natural language and on spatial clues.

Systems to analyze documents are commercially available. For instance, Textbridge (http://www.scansoft.com/textbridge) correctly handles most newspaper pages where articles are divided into blocks, but where blocks of the same article are spatially contiguous. The algorithms we propose could extend such a system to perform understanding of newspaper pages with articles whose blocks are scattered on one page.

The results presented in this article open a number of issues for future investigation. First, one may want to consider larger newspaper collections in languages other than Italian. The techniques used are independent of the Italian language and our implementation can be easily ported to other languages. Second, one may attempt to consider all the articles in the same newspaper, not only on the same page. It is often the case (especially on the first page) that the same article goes from one page to one or more following ones. Third, one may investigate the result of combining text processing with spatial clues to perform article clustering.

We have shown how article clustering can be effectively performed using text processing techniques and in particular we may conclude, as it has been often concluded in dealing with Information Retrieval problems, that 'simple is beautiful'. The simple clustering algorithm after removing stop words has high performance rate and a low computational complexity.

\section{Acknowledgments}

We thank the anonymous reviewers for suggestions and comments which greatly improved the presentation of the paper; Gabriel Kuper and Maciej Michno for careful proofreading; Enrico Blanzieri, Bernardo Magnini, Christof Monz, and Emanuele Pianta for fruitful discussion on the topic; in addition, Emanuele offered valuable support with the tools described in [Tonella et al., 2004]. Andrea Pegoretti thanks the Department of Information and Communication Technologies of the University of Trento for the support while completing his 'Tesi di Laurea Triennale' in Computer Science.

\section{References}

[Aiello et al., 2002] Aiello, M., Monz, C., Todoran, L., and Worring, M. (2002). Document Understanding for a Broad Class of Documents. International Journal of Document Anal- 
ysis and Recognition IJDAR, 5(1):1-16.

[Aiello and Smeulders, 2004] Aiello, M. and Smeulders, A. (2004). Thick 2D Relations for Document Understanding. Information Sciences, 167(3-4):147-176.

[Allen, 1983] Allen, J. (1983). Maintaining knowledge about temporal intervals. Communications of the ACM, 26:832-843.

[Baeza-Yates and Ribeiro-Neto, 1999] Baeza-Yates, R. and Ribeiro-Neto, B. (1999). Modern Information Retrieval. Addison Wesley.

[Balbiani et al., 1998] Balbiani, P., Condotta, J., and Fariñas del Cerro, L. (1998). A model for reasoning about bidimensional temporal relations. In Cohn, A., Schubert, L., and Shapiro, S., editors, Proceedings of the 6th International Conference on Principles of Knowledge Representation and Reasoning (KR'98), pages 124-130. Morgan Kaufmann.

[Cesarini et al., 1998] Cesarini, F., Gori, M., Marinai, S., and Soda, G. (1998). Informys: A flexible invoice-like form-reader system. IEEE Transactions on PAMI, 20(7):730-746.

[Dengel and Dubiel, 1997] Dengel, A. and Dubiel, F. (1997). Logical labeling of document images based on form layout features. In Proceedings of the 1997 Workshop on Document Image Analysis, DIA'97, pages 26-31, Ulm, Germany.

[Klink et al., 2000] Klink, S., Dengel, A., and Kieninger, T. (2000). Document structure analysis based on layout and textual features. In Proceedings of Fourth IAPR International Workshop on Document Analysis Systems, DAS2000, pages 99-111, Rio de Janeiro, Brazil.

[Lee et al., 2000] Lee, K., Choy, Y., and Cho, S. (2000). Geometric structure analsysis of document images: A knowledge approach. IEEE Transactions on PAMI, 22(11):12241240.

[Li and Ng, 1999] Li, X. and Ng, P. (1999). A document classification an extraction system with learning ability. In ICDAR'99, pages 197-200, Bangalore, India.

[Palmero and Dimitriadis, 1999] Palmero, G. S. and Dimitriadis, Y. (1999). Structured document labeling and rule extraction using a new recurent fuzzy-neural system. In ICDAR'99, pages 181-184, Bangalore, India.

[Pegoretti, 2004] Pegoretti, A. (2004). Clustering of article blocks in newspaper pages. Master's thesis, Univ. of Trento.

[Salton and McGill, 1983] Salton, G. and McGill, M. J. (1983). Introduction to Modern Information Retrieval. McGraw-Hill. 
[Sparck Jones, 1972] Sparck Jones, K. (1972). A statistical interpretation of term specificity and its application in retrieval. Journal of Documentation, 28(1):11-21.

[Tonella et al., 2004] Tonella, P., Ricca, F., Pianta, E., and Girardi, C. (2004). Using keyword extraction for web site clustering". In Proc. of WSE 2003, 5th International Workshop on Web Site Evolution.

[Tsujimoto and Asada, 1992] Tsujimoto, S. and Asada, H. (1992). Major Components of a Complete Text Reading System. Proceedings of the IEEE, 80(7):1133-1149.

[Walischewski, 1997] Walischewski, H. (1997). Automatic knowledge acquisition for spatial document interpretation. In Proceedings of the 4th ICDAR'97, pages 243-247, Ulm, Germany. 